\title{
Respuesta de material genético de Paulownia spp a tratamiento silvicultural, como estrategia para evaluar su adaptabilidad a condiciones climáticas de estepa espinosa Montano Bajo, Ecuador
}

Corresponding Author:

R. A. Ramos Veintimilla

raul.ramos@espoch.edu.ec

Received: 10 January 2020

Accepted: 17 January 2020

Published: 26 January 2020

Publishing services provided by Knowledge E

(c) R. A. Ramos Veintimilla et al. This article is distributed under the terms of the Creative Commons Attribution License, which permits unrestricted use and redistribution provided that the original author and source are credited.

Selection and Peer-review under the responsibility of the $\mathrm{VI}$ Congreso Internacional Sectei 2019 Conference Committee.

\section{S OPEN ACCESS}

\section{Response of Paulownia spp genetic material to silviculture, as a strategy to assess its adaptability to the climatic conditions of thorny steppe Montano Bajo, Ecuador}

R. A. Ramos Veintimilla1, A. S. Guanga Paredes², F. A. Sigcha ${ }^{3}$, and F. M. Romero Mancero ${ }^{4}$

${ }^{1}$ Docente de Silvicultura, Escuela de Ingeniería Forestal, Facultad de Recursos Naturales, Escuela Superior Politécnica de Chimborazo. Panamericana Sur km 11\%2. Riobamba, Ecuador. EC060155 ${ }^{2}$ Escuela de Ingeniería Forestal, Facultad de Recursos Naturales, Escuela Superior Politécnica de Chimborazo

${ }^{3}$ Estación Experimental Santa Catalina, Instituto Nacional de Investigaciones Agropecuarias. Responsable programa Forestería

${ }^{4}$ Agencia Ecuatoriana de Aseguramiento de la Calidad del Agro, Distrito 3 Riobamba

\section{Resumen}

El propósito de la investigación consistió en estudiar la respuesta de Paulownia elongata, $P$. fortunei y un híbrido (Paulownia fortunei $x$ elongata) a un sistema de recepa y evaluar el desempeño de los brotes juveniles, como estrategia para confirmar su adaptabilidad a las condiciones bioclimáticas de estepa espinosa Montano Bajo. EI ensayo se instaló en diciembre del 2 015, en una plantación clonal de dos años de edad, plantada por INIAP y ESPOCH en la granja Tunshi, Ecuador. Se establecieron 9 bloques por especie para un total de 27 parcelas experimentales, con 9 observaciones por parcela neta. El tratamiento silvicultural ejecutado fue poda de renovación/recepa, realizando un corte del tallo en bisel a dos centímetros de altura desde el suelo. Las variables evaluadas fueron: Días a la brotación, número de brotes por tocón, diámetro a la base del brote y altura del brote. Los tres materiales estudiados respondieron positivamente a la práctica silvicultural, $90 \%$ de tocones brotados a los 61 días de haber realizado el ensayo. A los 29 días de la recepa ya se encontraron brotes epicórmicos en las tres especies de Paulownia, presentando entre 1 y 13 brotes por tocón. En crecimiento dasométrico, $P$. fortunei experimentó el mayor promedio en altura $(81,85 \mathrm{~cm})$, seguida por $P$. elongata $(70,51 \mathrm{~cm})$ y Paulownia hibrido $(65,53 \mathrm{~cm})$, siendo 3,5 veces mayor a los promedios de altura y 4 veces mayor en diámetro a la altura de la base del tallo, de los reportados en el primer año de evaluación. Estos resultados avizoran probables comportamientos adecuados y adaptación como 
una especie forestal-agroforestal de interés para características similares del sitio de estudio.

Abstract: This study is aimed to evaluate the response Paulownia elongata, $P$. fortunei and hibrid (Paulownia fortunei $x$ elongata) to renewal pruning system and evaluate to develop the juvenile sprout of plants as a strategy to assess its adaptability to the bioclimatic conditions of thorny steppe Montano Bajo, Ecuador. The essay was install in December, 2015 in one clonal plantation two years old, establish by INIAP and ESPOCH in Tunshi farm, Ecuador. This was established in nine blocks per species for a total of 27 experimental plots with nine observations per net plot. The selviculture treatment executed was renewal pruning, making a bevel cut stem two feet high from the ground. The variables evaluated were: Days sprouting, number of sprouts per stump diameter at the base of the outbreak and height of the outbreak. The three materials studied responded positively to silvicultural practice and $90 \%$ of erupted stumps at 61 days responded to development essay. At 29 days from the development the recepa presented epicormics sprout in the three Paulownia species; $P$. fortunei experienced the greatest average height $(81,85 \mathrm{~cm})$, followed by $P$. elongata $(70,51 \mathrm{~cm})$ and hybrid Paulownia $(65,53 \mathrm{~cm})$, being 3,5 times greater than the average height and 4 times greater in diameter at the base than those reported in the first year evaluation, these results foresee probable behaviors adapted and adequate as a forestry-agroforestry species of interest from the site of study

Palabras clave: recepa, poda, silvicultura, dendroenergía

Keywords: recepa, pruning, silviculture, wood energy

\section{Introducción}

La deforestación y degradación de los bosques, constituyen dos de los mayores problemas ambientales a nivel mundial. Las estadísticas forestales en el Ecuador revelan 9 599 678,7 hectáreas de bosques existentes (34,7 \% de la superficie nacional), de los cuales el $98,5 \%$ son bosques naturales, en tanto que las plantaciones no superan el $1,5 \%$ restante del patrimonio forestal (1). Estas cifras sumadas y comparadas con el uso potencial del suelo, sugieren que en el país existe un déficit de cobertura forestal de aproximadamente 2,0 a 2,5 millones de hectáreas $(1,2)$. 
Esa reducción sistemática de los bosques nativos, ha sido provocada por una irracional explotación de los recursos forestales para distintos fines y usos, pero sobre todo para la industria de la madera, siendo el proceso de colonización, el eje principal mediante el cual se evidenció transferencia de tierras para uso agropecuario en detrimento de tierras con aptitud forestal (3).

En el país, tradicionalmente se viene explotando plantaciones de especies forestales de rápido crecimiento, introducidas y nativas, entre estas algunas especies de eucalipto (Eucalyptus globulus Labill., E. urophylla S.T. Blake X E, E. grandis W. Mill ex Maiden, E. saligna Sm.), pachaco (Schizolobium parahiba Vell. S.F. Blake), melina (Gmelina arborea Roxb.), laurel (Cordia alliodora Ruiz \& Pav.), pinos (Pinus radiata D. Don, P. patula Schltdl. \& Cham), y balsa (Ochroma pyramidale Cav. ex Lam. Urb.), sin embargo, el aprovechamiento intensivo de estas especies ha provocado pérdida de su reducida base genética, cuyos individuos sobresalientes se han tornado cada vez más escasos para cubrir la demanda interna y externa de madera de aserrío, industrial o energía de biomasa $(4,5,2,6,7,8,9,10,11)$. Lo indicado, ha obligado a agendar la necesidad de buscar nuevas especies forestales con atributos de crecimiento rápido, como potenciales alternativos para contribuir a las crecientes demandas forestales del país.

Paulownia es un género forestal perteneciente a la familia Paulowniaceae (12). Nueve especies de este género son originarias de China, a excepción de $P$. fortunei (Seem.) Hemsl, que se extienden hasta Vietnam y Laos, mientras que $P$. tomentosa (Thunb.) Steud crece en Corea y Japón (13). De estas, las especies más utilizadas para proyectos forestales son $P$. elongata S. Y. Hu, P. fortunei y P. kawakamii T. Itô en razón de sus características de crecimiento rápido (14). Son árboles que se cultivan hace más de 2600 años, pero que empezaron a ser estudiados a partir de 1972 por el investigador forestal de origen chino (13, 14). Estos árboles empezaron a ser desarrollados genéticamente a comienzos de la década de 1 990, mediante la evaluación de su adaptación a distintos climas a fin de promover su cultivo en el mundo, tanto para reforestación como para uso maderable y energético. Actualmente presenta una amplia distribución, desde el este de Asia --principalmente en Japón y Corea, pasando por Indonesia, Estados Unidos (Carolina del Norte y del Sur, California, Indiana y Kentucky) e India, hasta México y Brasil en América Latina $(15,16)$.

En sitios adecuados los árboles presentan gran porte, fuste recto, cilíndrico, de color grisáceo, con suaves estrías longitudinales y casi nunca presentan nudos; un árbol de diez años de edad puede alcanzar volúmenes aproximados de 4, 0-4,5 $\mathrm{m}^{3}$, con crecimientos anuales en DAP de 3 a $4 \mathrm{~cm}(13,14)$. Tales atributos, se manifiestan en la 
excelente calidad y belleza de su madera, su considerable producción de biomasa y capacidad de fijación de $\mathrm{CO}_{2}$, potencial de aprovechamiento del follaje para el ganado, y también para programas de reforestación de tierras abandonadas y/o degradadas, además de sus atributos ornamentales (16). Paulownia sp, se adaptan a una gran variedad de climas, llegando a soportar mínimas absolutas de $-20{ }^{\circ} \mathrm{C}$ y máximas absolutas de $45{ }^{\circ} \mathrm{C}$, su rango óptimo de temperaturas se encuentra entre $24{ }^{\circ} \mathrm{C}$ y 29 ${ }^{\circ} \mathrm{C}$ de temperatura media diaria (13). En relación a la altitud, el rango que normalmente ocupa esta especie varía entre los 600 y 1500 metros sobre el nivel del mar (17).

La Presidencia de la República del Ecuador en enero 2013 manifestó el interés por explorar la introducción y evaluación de la adaptación de especies forestales foráneas con atributos de Crecimiento Rápido, particularmente por especies del género Paulownia spp, como potenciales alternativas para contribuir a las prioridades políticas contempladas en el Plan Nacional de Forestación y Reforestación Productiva (4). Por lo cual el INIAP auspiciado por la SENESCYT, en mayo del 2 013, inició el proyecto de investigación "Adaptación de especies forestales de rápido crecimiento del género Paulownia spp a diversos ambientes bioclimáticos y suelos del Ecuador", donde uno de los sitios de investigación del proyecto fue estepa espinosa Montano Bajo (Granja Experimental Tunshi-ESPOCH), en éste sitio, en su evaluación inicial se observó una defoliación total de las plantas, asociado con una pérdida de dominancia apical y posterior crecimiento vigoroso de yemas axilares, características indeseadas para árboles de producción de madera sólida. Por este motivo se desarrolló el presente estudio, que tiene como objetivo estudiar la respuesta de tres especies forestales del genero Paulownia spp a podas de renovación y comportamiento de los brotes juveniles, como estrategia para evaluar y confirmar su adaptabilidad a las condiciones bioclimáticas de estepa espinosa Montano Bajo.

\section{Materiales y Métodos}

\subsection{Descripción del sitio}

El estudio se llevó a cabo entre diciembre del 2015 y marzo del 2 016, en un experimento plantado por el INIAP-ESPOCH en diciembre del 2014, en la granja experimental Tunshi de la ESPOCH, ubicada en el km 4 de la vía Riobamba-Licto con coordenadas proyectadas UTM $\mathbf{X}=671167,34 \mathrm{E}, \mathbf{Y}=9878372,68 \mathrm{~N}$. La temperatura media anual es de $13,8{ }^{\circ} \mathrm{C}$ con una precipitación media anual de $835,6 \mathrm{~mm}$, la altitud es de $2700 \mathrm{msnm}$. El sitio corresponde a estepa espinosa Montano Bajo (18). 


\subsection{Descripción del ensayo}

Las especies estudiadas fueron Paulownia elongata, $P$. fortunei y el hibrido (P. elongata

* P. fortunei), introducidas desde los Estados Unidos de Norte América y establecidas en 9 bloques por especie para un total de 27 parcelas experimentales, con 9 observaciones por parcela neta. El tratamiento silvicultural consistió en realizar una poda de renovación, realizando un corte inclinado/corte en bisel a dos centímetros de altura desde el suelo a cada uno de los arbolitos que comprenden cada una de las parcelas netas $(n=9)$, posterior a la poda se aplicó con una brocha una pasta de oxicloruro de $\mathrm{Cu}$ en el corte, para evitar el ingreso de patógenos y se etiquetaron cada uno de los individuos en estudio, para poder diferenciarlos entre ellos y registrar la información. Posteriormente se realizó un raleo de rebrotes, seleccionando el rebrote dominante, basándose en características morfológicas como fuste recto, vigoroso, sin daños mecánicos y sin incidencias de plagas. El número de rebrotes que se dejaron en el tocón fue uno, tomando en cuenta el diámetro y distribución en éste.

\subsection{Metodología de toma de datos}

Las variables evaluadas fueron: Días a la brotación, esta evaluación fue visual determinando la presencia de los rebrotes en cada uno de los tocones y se reportó en porcentaje de tocones brotados a la fecha de evaluación. Sobrevivencia de rebrotes. La sobrevivencia se evaluó en base al número de rebrotes vivos que se observaron a los 61 días de realizado la poda, utilizando la metodología de $(19,20)$, quien indica que un porcentaje de sobrevivencia superior al 80\% corresponde a categoría buena, entre 40 y $80 \%$ de sobrevivencia corresponde a categoría regular, y porcentaje de sobrevivencia menos de 40 por ciento corresponde a categoría mala. Número de rebrotes por tocón, mediante observación directa se registraron el número de brotes presentes en el tocón (en cada individuo podado) y se reportó en promedio de brotes por unidad de observación. Diámetro a la base del rebrote, esta variable se registró a partir de que el brote alcanzó $15 \mathrm{~cm}$ de altura y fue seleccionado presentando las mejores características morfológicas; para asegurarse que todas las mediciones se realicen en el mismo sitio se ejecutó una marca con pintura, a dos centímetros de la inserción del rebrote en el tocón y con una forcípula graduada se midió en dos direcciones (N-S y E-W) el diámetro de éste (Fig. 1), sus datos se registraron en centímetros. Altura del rebrote/eje, se registró el crecimiento del rebrote midiendo desde la inserción en el tocón hasta la yema terminal a lo largo del fuste (21) para lo cual se utilizó una cinta 
métrica graduada en centímetros (Fig. 2), y se reportó en la misma unidad. Todas estas variables se evaluaron con una frecuencia de 8 días a partir de los 15 días de haber realizado la poda. Los datos se analizaron, dependiendo de la variable, usando medidas de tendencia central y análisis de medidas repetidas en el tiempo para las variables dasométricas, así como separación de medias Duncan al 5\%, en el programa InfoStat ${ }^{\circledR}$ versión 2014.

\section{Resultados y discusión}

\subsection{Días a la brotación y sobrevivencia del rebrote}

El análisis estadístico realizado para porcentaje de brotación de Paulownia como efecto a la poda de restauración, no encontró diferencias estadísticas para las especies en estudio en ninguna de las fechas evaluadas. Sin embargo, la Fig. (1) indica los porcentajes de brotación en los diferentes períodos, es así que las tres especies en estudio se encontraron rebrotadas alrededor del 55\% de tocones a los 14 días de realizado la poda de restauración, porcentajes que se fueron incrementando hasta los 45 días de haber realizado dicha práctica silvicultural alcanzando entre el 78 y $87 \%$ de tocones rebrotados, a los 61 días después de la poda en las tres especies se encontró más del 90 \% de tocones rebrotados. Aunque las diferencias son numéricas, se observa cierta superioridad en la brotación en $P$. fortunei, respuesta que probablemente está relacionada con el diámetro del tocón de la planta podada, ya que esta especie presentó el 90 \% de los individuos un diámetro del tocón superior a $3 \mathrm{~cm}$, mientras que las otras especies presentaron el $80 \%$ de los individuos con éstas características. Junto a la figura 1 se observa una fotografía de un tocón de $P$. fortunei a los 29 días después de la poda. Aunque la literatura reporta estudios extremadamente limitados en recepa de Paulownia, los resultados de brotación y sobrevivencia encontrados en esta investigación corresponden a la categoría de buenos (19) quien indica que un porcentaje de sobrevivencia superior al 80\% corresponde a categoría buena, entre 40 y $80 \%$ de sobrevivencia corresponde a categoría regular, y porcentaje de sobrevivencia menos de 40 por ciento corresponde a categoría mala; así como también $(21,22)$ en un estudio de rebrotes en Eucalyptus camaldulensis Dehnh reportaron sobrevivencia del $88 \%$. Otros investigadores (23), reportan valores mayores (57,34 \%) de mortalidad de rebrote, en un estudio de la capacidad de rebrote de Leucaena macrophyla Benth, valor considerado como aceptable según menciona (24) para las especies de género Leucaena sp.; por su parte (25) en un estudio del crecimiento y rendimiento de biomasa 
aérea de rebrotes de dos años en cultivos dendroenergéticos, reportaron sobrevievencia de $84 \%$ para E. globulus, 60 \% para E. denticulata y $30 \%$ para Acacia delabata. La mortalidad de rebrotes se debe probablemente a que la planta al ser podada entra en estrés fisiológico lo que tiende a generar nuevos ejes en mayor cantidad, con el fin de restablecer los procesos de fotosíntesis y transpiración. Una vez generada la nueva cobertura foliar, tiende a eliminar los ejes con menor grado de desarrollo o de menor aporte energético (la generación de azúcares por producto de la fotosíntesis es baja), lo cual incide que la mortalidad aumente de un 40 a 60 \% (26).

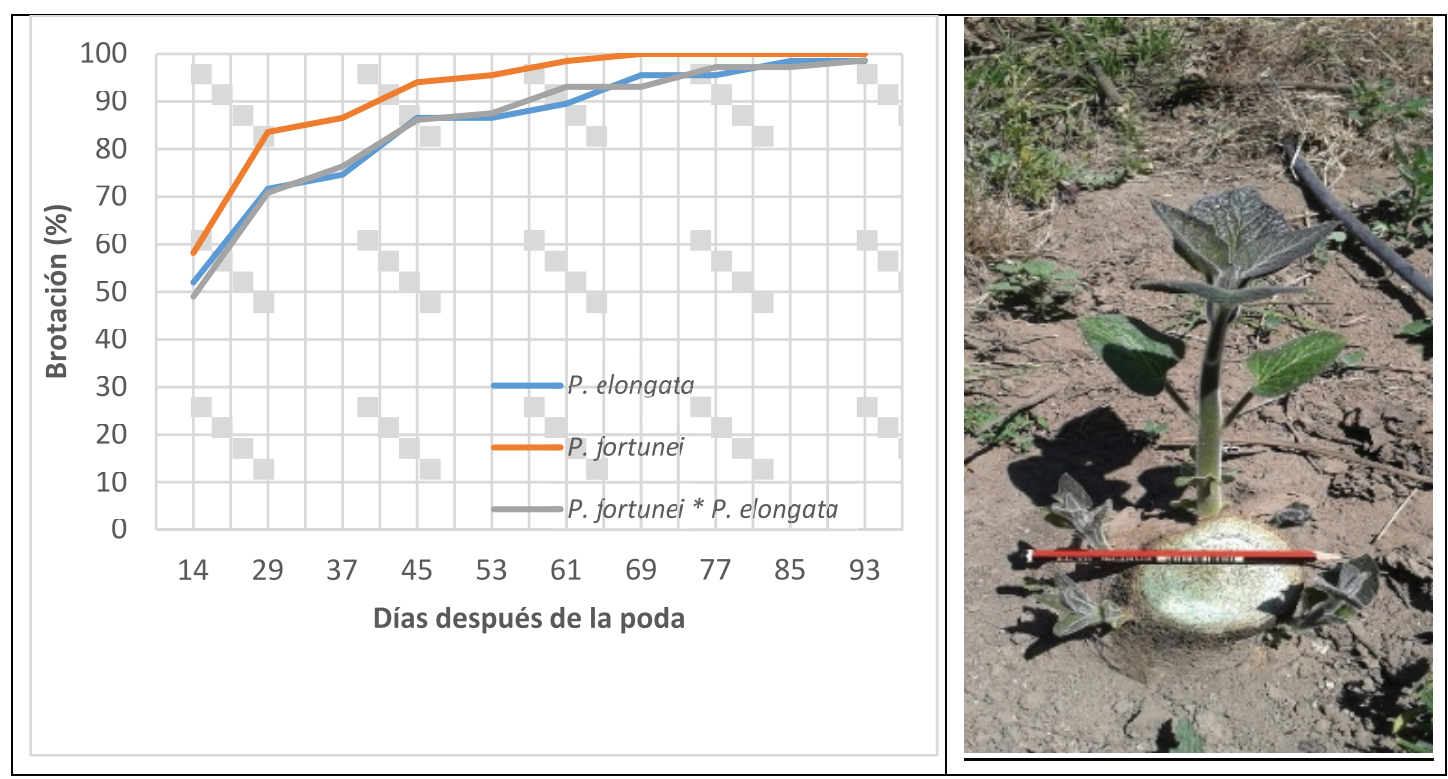

Figure 1: Porcentaje de brotación en tocones durante los primeros 93 días de evaluación de Paulownia sp (P. elongata, P. fortunei e hibrido, P. elongata ${ }^{*} P$. fortunei). Tunshi-ESPOCH.

\subsection{Número de brotes por tocón}

A partir de los 14 días de realizado la poda de restauración ya se encontraron brotes epicórmicos en las tres especies de Paulownia estudiadas (Fig. 1), mismos que presentaron entre 1 y 9 brotes por tocón (Tabla 1), alcanzando su número máximo de brotes a los 29 días de haber realizado la práctica silvicultural, de éstas la especie que mayor número de brotes presentó fue el Hibrido ( $P$. elongata $\times$. fortunei) alcanzando un número entre 1 y 13 (Tabla 1). La capacidad de rebrote de las especies estudiadas se encuentra categorizada como excelente para Paulownia híbrido y bueno para $P$. fortunei y $P$. elongata (27) excelente corresponde a 10 o más rebrotes, bueno de 5 a 9,5 rebrotes, aceptable de 3 a 4,9 rebrotes y malo menos de 2,9 rebrotes. En estudios similares en Leucaena macrophyla Benth (23), reportaron promedios de 1,78 a 4,68 rebrotes/planta, evaluado en estación lluviosa y seca; datos similares reportan (28) con 
especies arbóreas perennes tropicales, encontrando que un aumento de los rebrotes en época seca es una respuesta mecánica al estrés hídrico que presenta la planta. Así como también (29) en un estudio de la capacidad de rebrote en clones hibridos de Populus encontraro entre 12 y 13,5 rebrotes / planta y Un árbol podado al no tener copa en presencia de déficit hídrico, debe desarrollar una nueva cobertura foliar que permita generación de azúcares y el intercambio gaseoso para la sobrevivencia del individuo.

TABLE 1: Rango de número de brotes por tocón de Paulownia sp. Tunshi-ESPOCH.

\begin{tabular}{l|c|c|c|c|}
\hline Especie & \multicolumn{4}{|c|}{ Días después de la poda de restauración } \\
\hline & $\mathbf{1 4}$ días & $\mathbf{2 9}$ días & $\mathbf{3 7}$ días & $\mathbf{4 5}$ días \\
\hline P. elongata & $1-7$ & $1-9$ & $1-9$ & $1-9$ \\
\hline P. fortunei & $1-8$ & $1-8$ & $1-8$ & $1-8$ \\
\hline Paulownia Hibrido & $1-9$ & $1-13$ & $1-13$ & $1-13$ \\
\hline
\end{tabular}

\subsection{Altura del brote}

El análisis estadístico realizado para altura del brote identificó diferencias altamente significativas ( $p$-valor $=0,0008$ ) para la interacción "especies * tiempo de evaluación". En la Fig. (2) se muestran las tendencias del crecimiento de las tres especies de Paulownia en estudio durante los 93 días de evaluación y su respectiva separación de medias. Donde se puede observar que Duncan al 5\% agrupa a las alturas del brote en varios rangos de clasificación, identificando a $P$. fortunei como la especie de mayor crecimiento promedio $(81,85 \mathrm{~cm})$, seguida por $P$. elongata $(70,51 \mathrm{~cm})$ y $P$. fortunei * P. elongata $(65,53 \mathrm{~cm})$. Estos crecimientos experimentados en las tres especies, después de los 60 días de haber iniciado la brotación fueron 3,5 veces mayor a los promedios de altura de planta reportados por (30) en un período similar en el primer año de evaluación. Las especies evaluadas han presentado crecimientos nunca reportados para especies forestales en la zona de estudio. Los datos encontrados en esta investigación superan en por lo menos el 50 \% a los reportados por (24) en un estudio del crecimiento y rendimiento de biomasa aérea de rebrotes en cultivos dendroenergéticos, presentando alturas del rebrote de $230 \mathrm{~cm}$ para E. globulus, 180 $\mathrm{cm}$ para E. denticulata y $140 \mathrm{~cm}$ para Acacia delabata en dos años de edad; así como también, al promedio de $50 \mathrm{~cm}$ de altura de planta de Leucaena macrophyla Benth, reportado por (23) y promedios de 117,28 cm de altura para Lonchocarpus minimiflorus durante los 6 meses de evaluación 


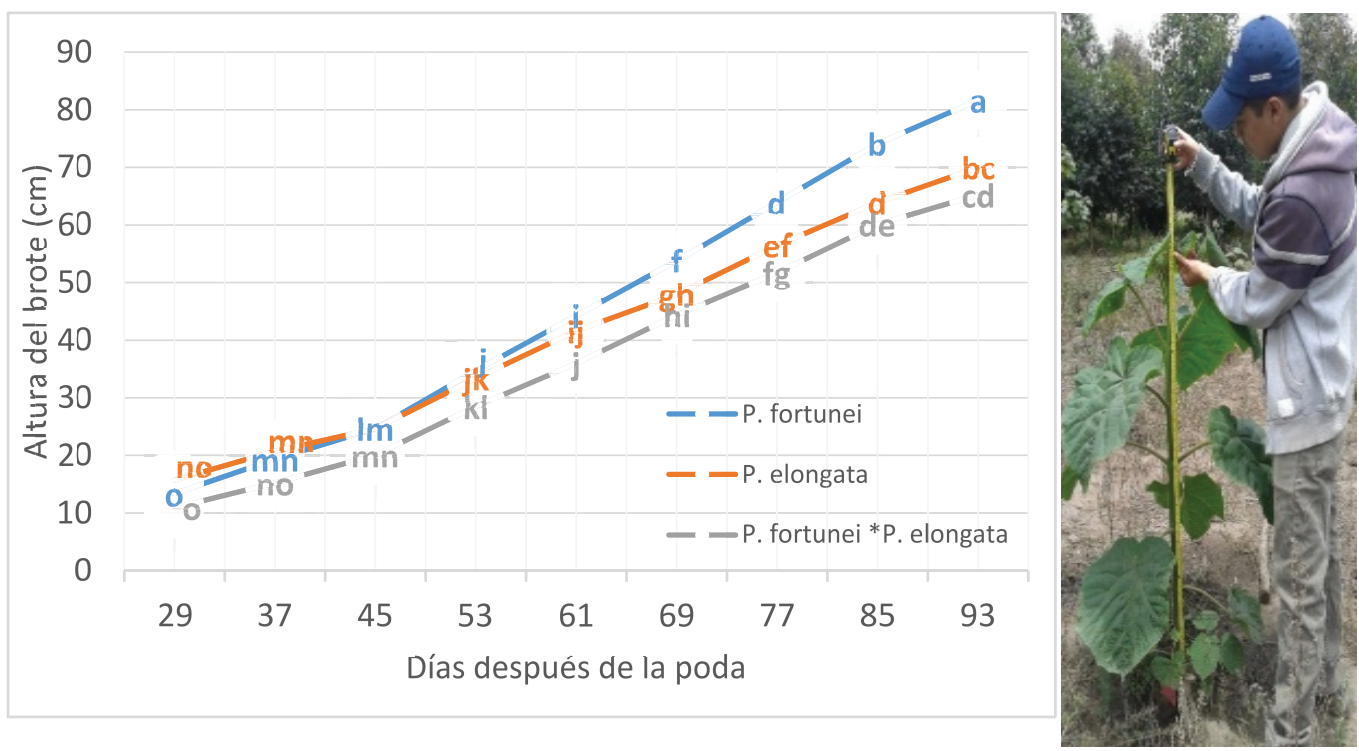

Figure 2: Crecimiento semanal de altura del brote de $P$. fortunei, $P$. elongata y $P$. fortunei ${ }^{*} P$. elongata bajo el efecto de poda de renovación. Tunshi-ESPOCH (Duncan 5\%).

\subsection{Diámetro a la base del brote}

El diámetro del brote a la base del eje-tallo (dos centímetros de la inserción del tocón), en las especies en estudio, experimentaron tendencias similares a la variable altura del brote, mostrando diferencias estadísticas significativas $(p<0,05)$ para especies en el tiempo evaluado, dicha diferencia se observa con mayor claridad a partir de los 69 días de haber realizado la poda de restauración. En la Fig. (3) se muestran las tendencias de crecimiento del diámetro a la base para las tres especies durante los 93 días de evaluación, donde se puede observar que $P$. fortunei presenta el mayor promedio 2,2 cm de Incremento Medio Semanal (IMS), seguido por P. elongata (1,95 $\mathrm{cm})$ y finalmente $P$. fortunei ${ }^{*} P$. elongata $(1,85 \mathrm{~cm})$. Al igual que en la variable altura del brote, probablemente las especies están respondiendo a la adaptación en el sitio, en razón de que luego de la práctica silvicultural las especies presentaron un diámetro a la base del eje 4 veces superior al experimentado en el primer año de establecimiento del experimento, durante un mismo período de comparación (30).

Esta mayor vigorosidad en altura y en diámetro a la base del tallo se explica por la menor competencia al existir un solo rebrote seleccionado, lo que significa que, si continúa este comportamiento, probablemente a una misma edad de rotación este tratamiento obtendrá un producto de mayores dimensiones comerciales, tendencia similar manifiesta (31) en su investigación "Monte Bajo, opción para tres especies de Eucaliptos en segunda rotación, un caso en la provincia de Osorno, Décima Región, Chile". Así como también (23) en un estudio de rebrote de Leucaena macrophyla 
Benth, reportan un crecimiento diamétrico de 0,12 cm por semana, y (32) reportan crecimientos de $0,13 \mathrm{~cm}$ por semana en la especie Lonchocarpus minimiflorus promedio experimentado en una investigación de la capacidad de rebrote de especies arbóreas del bosque seco Secundario de Nandarola, Nandaime, Granada (33)

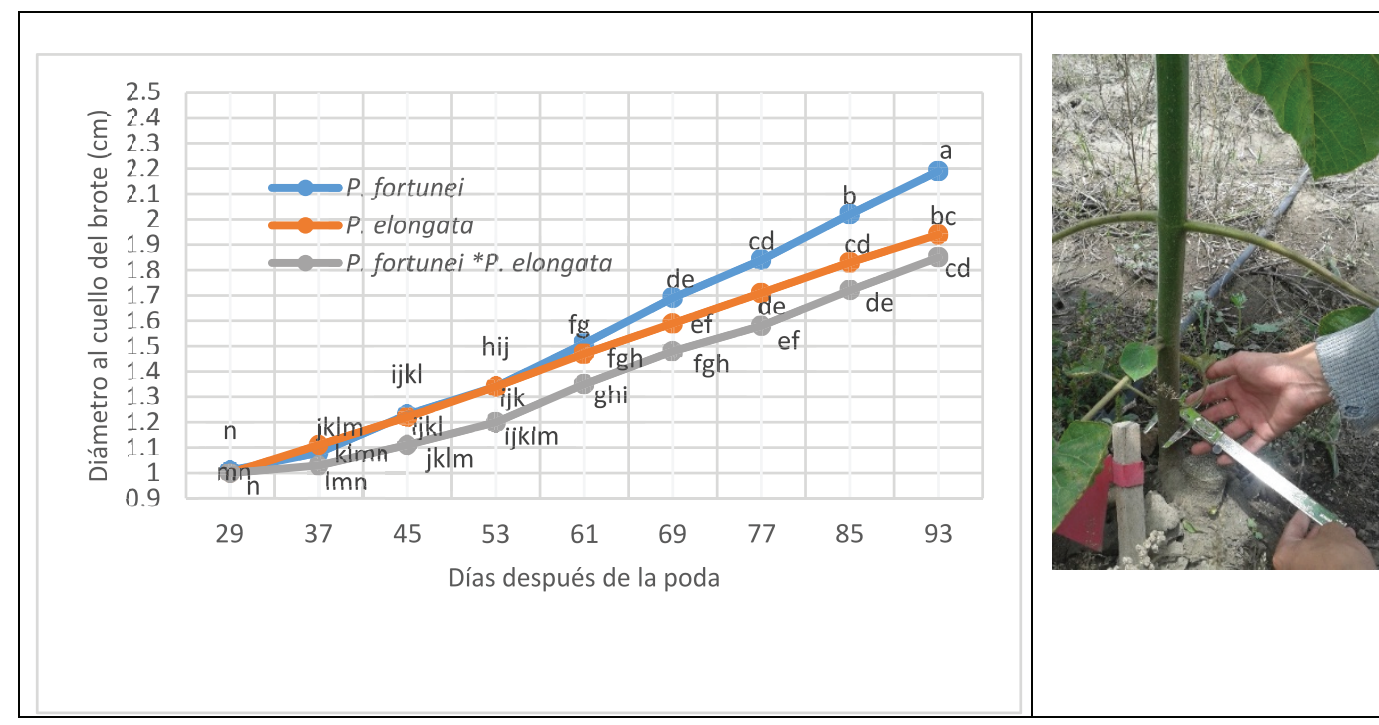

Figure 3: Crecimiento semanal de diámetro a la base del brote de $P$. fortunei, $P$. elongata y $P$. fortunei ${ }^{*} P$. elongata bajo el efecto de poda de renovación. Tunshi-ESPOCH (Duncan 5\%).

\section{Comparativo de variables de crecimiento dasométrico de Paulow- nia spp entre sitios experimentales de Ecuador.}

En la Fig. (4) se presentan los datos de variables de crecimiento dasométrico como son: Altura del eje/planta en cm (Figura 4A) y diámetro a la base del eje en mm (Figura 4B) de tres especies de Paulownia evaluadas en dos sitios contrastantes de Ecuador (Quevedo = bosque húmedo Tropical, y Riobamba = estepa espinosa Montano Bajo) (31); adicionalmente se hace una comparación del comportamiento de dichas variables y especies forestales en el sitio Riobamba, con la particularidad de que la segunda evaluación se realiza dos años después de la plantación, luego de haber aplicado una práctica silvicultural (poda de renovación), como una estrategia para reconfirmar su adaptación de las especies al sitio.

La altura promedio del eje de Paulownia experimentado en el sitio Quevedo, 60 días después de la plantación (33), fue parecida a la expresada en Riobamba 60 días después de la brotación, posterior a la poda de renovación practicada $(74 \mathrm{~cm})$, y ésta fue 2,5 veces superior al crecimiento promedio presentado en el sitio Riobamba 60 días después de la plantación; se puede observar también que el crecimiento promedio en 
altura en los 60 días después de la brotación fue ligeramente superior al crecimiento en el mismo ensayo a los 12 meses de plantado (Fig. 4A).

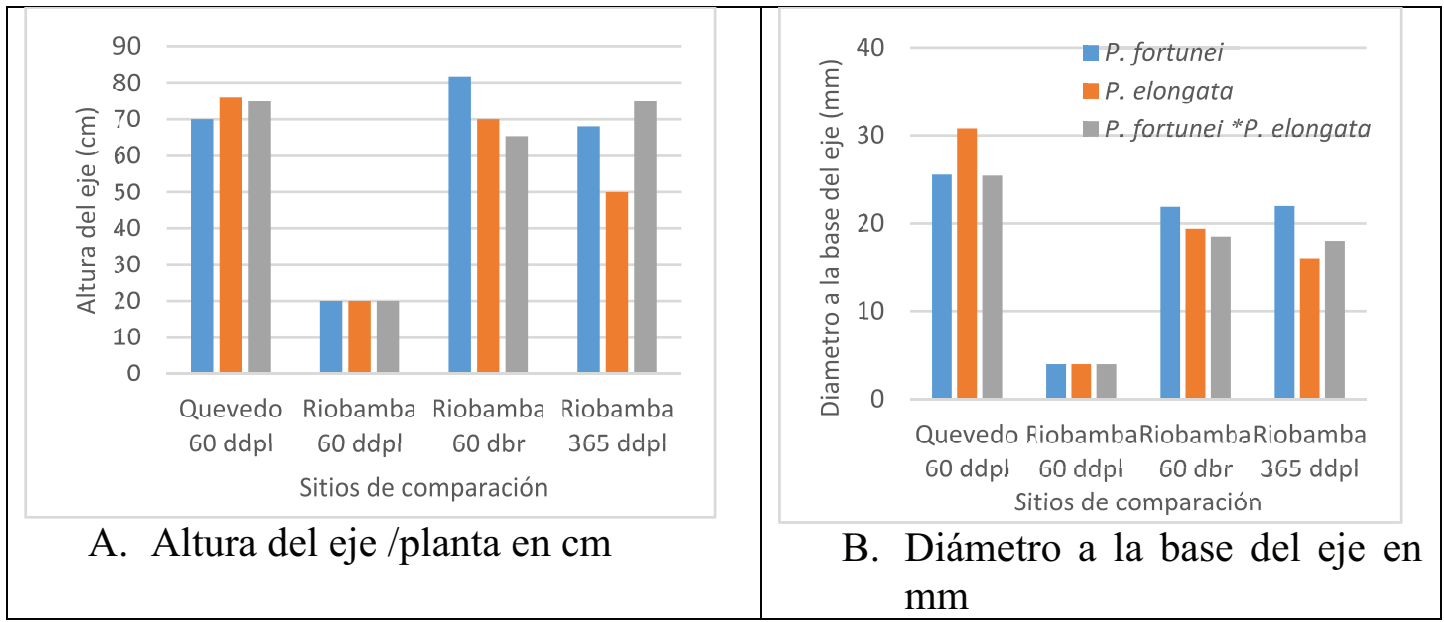

Figure 4: Comportamiento de variables dasométricas de $P$. fortunei, $P$. elongata y $P$. fortunei * $P$. elongato de sitios experimentales en Ecuador (Datos tomados de Guilcapi, 2015 y Guzmán, 2015. 60 ddpl = 60 días después de la plantación y $\mathbf{6 0} \mathbf{d d r}=60$ días después de haber rebrotado).

Para la variable diámetro a la base del eje (Fig. 4B), se observa tendencias similares a las experimentadas en altura del eje/planta, e inclusive al comparar los datos registrados en el sitio de Riobamba, el promedio a los 60 días después de la poda de restauración fue 4 veces superior a los promedios expresados a los 60 días después de la plantación (4 mm en 60 días después de la plantación Vs 20 mm 60 días después de rebrotado).

El comportamiento de las variables de crecimiento dasométrico experimentado por las tres especies estudiadas en el sitio estepa espinosa Montano Bajo, luego de la práctica silvicultural (poda de restauración) advierte un grado interesante de adaptación de las especies al sitio (suelo, clima), sin embargo no es un tiempo suficiente de evaluación para llegar a conclusiones concretas, por lo que hay la necesidad de seguir evaluándoles a los brotes por lo menos los 9 meses siguientes, y con ésos datos llegar a conclusiones más confiables.

\section{Conclusiones}

- Bajo las condiciones de sitio (clima y suelo) estepa espinosa Montano Bajo, las tres especies de Paulownia respondieron positivamente a la poda de renovación, en razón de que, a los 61 días de haber podado, en las tres especies se encontró más del 90 \% de tocones brotados, esto nos indica la capacidad de rebrote de las especies y por tanto el potencial que demuestran para programas de clonación, aseveración que lo ratifica la literatura en varias publicaciones. 
- Al comparar el comportamiento de las variables dasométricas a los 60 días después de la práctica silvicultural y al año de plantación, sin la práctica silvicultural, se registró una superioridad en altura de 3.5 veces y en diámetro de 4 veces en favor de la poda de recepa, mostrándose ésta como un tratamiento silvicultural favorable para evaluar procesos de adaptación de especies latifoliadas como lo es Paulownia.

- El efecto de la práctica silvicultural sobre el comportamiento de las variables dasométricas de las tres especies de Paulownia evaluadas en estepa espinosa Montano Bajo, fue similar al efecto obtenido en bosque húmedo Tropical sin la práctica silvicultural en un período análogo de evaluación, lo que avizora probables comportamientos adecuados como una especie de interés para éstas características de sitio.

- Los datos de variables dasométricas registrados en los 93 días posteriores a la práctica silvicultural indicados en la presente investigación, muestran que las especies de Paulownia responden favorablemente a la poda de renovación en estepa espinosa Montano Bajo; a decir de los datos, son crecimientos nunca alcanzados por ninguna especie forestal en el sitio.

\section{Recomendaciones}

Investigar las posibles causas de la alta heterogeneidad en el crecimiento de los individuos de un mismo clon, poner mucha atención en las tres especies de Paulownia, sobre todo en $P$. fortunei y dar seguimiento de por lo menos dos períodos estacionales, donde los nuevos brotes reciban condiciones de estrés propias del sitio y sus respuestas permitan llegar a conclusiones convincentes sobre la adaptación de las mismas. Si continúan dichos comportamientos probablemente podrían ser especies de importancia socioeconómica y ambiental para las condiciones de sitio evaluadas.

\section{Agradecimientos}

Dejamos nuestro sincero agradecimiento al INIAP Santa Catalina por permitirnos desarrollar esta investigación en su ensayo de adaptación y a la Escuela de Ingeniería Forestal de la ESPOCH por aprobarnos y autorizar dicha investigación como un tema de práctica preprofesional de la actual Ing. Andrea Guanga. 


\section{Conflicto de intereses}

No existe ningún tipo de conflicto en razón de que la información generada en la investigación es para apoyo de quienes puedan necesitar en beneficio técnico científico.

\section{References}

[1] Carrión, D.; Chiu, M. 2011. Documento del Programa Nacional REDD. Sexta reunión de la Junta Normativa del Programa Nacional ANU- REDD. Disponible en: www. unredd.net/index.php?option=com_doeman\&task=doe.

[2] Grijalva, J.; X. Checa; R. Ramos; P. Barrera; R. Vera; F. Sigcha. 2016. Estado de los recursos genéticos forestales en Ecuador. Programa Nacional de Forestería del Instituto Nacional de Investigaciones Agropecuarias. INIAP, Quito. 100 p.

[3] FAO. 2010. Global Forest Resources Assesment 2010. Main Report. FAO. Disponible en: http//www.fao.org/docrep/013/i1757e/i1757e.pdf

[4] MAE. 2011. Programa de Naciones Unidas para la reducción de las emisiones por deforestación y degradación del bosque en los países en desarrollo Documento del programa nacional Conjunto. Sexta reunión de la junta normativa Del programa ONU-REDD. 21 - 22 de marzo De 2011. Da lat, Vietnam.

[5] Delgado, J.; P. Játiva. 2010. Políticas institucionales de Investigación, Transferencia de Innovaciones y Prestación de Servicios Tecnológicos, Quito, Ecuador INIAP, Dirección General, Dirección de Planificación y Economía Agrícola, 52 p. Publicación miscelánea No 154.

[6] Limongi, R.; G. Wiracocha; C. Yepez. 2011. Amarillo de Guayaquil (Centrolobium ocroxylum Rose ex Rudd) especie de uso múltiple del bosque seco del Ecuador. INIAP, Portoviejo. $32 \mathrm{p}$.

[7] Ministerio de Agricultura, Ganadería, Acuacultura y Pesca. 2013. Programa de incentivos para la reforestación con fines comerciales. Guayaquil, Ecuador

[8] Lascano, M. 2008. Valoración de la contribución forestal a la economía nacional, caso Ecuador. OTCA. Ecuador

[9] Palacios, W.; E. Vásquez; N. Jaramillo; M. Robalino. 2011. Evaluación de la estrategia de Desarrollo forestal Sustentable 2006-2011. Ministerio del Ambiente del Ecuador, Quito.66p.

[10] Prado, L.; C. Samaniego; J. Ugarte-Guerra. 2010. Estudio de las cadenas de abastecimiento de germoplasmas forestal en Ecuador. World Agroforestry Centre (ICRAF), Lima. 247 p. 
[11] Ramos Veintimilla, R. A.; A. M. Cárdenas Rubio; R. R. Vera Vélez; J. R. Limongi Andrade y J. E. Grijalva Olmedo. 2018. Propagación in vitro de tres especies del género Paulownia bajo el sistema de propagación convencional. Quebracho 25 (1,2):69-79. Argentina.

[12] García-Lahera, J. P. 2010. Guía de facilitación para el trabajo con la literatura de referencia sobre la flora de Cuba. Editorial Feijóo, Cuba.

[13] Zhu, Z. H.; C. J. Chao; X. Y. Lu; Y. G. Xiong. 1986. Paulownia in China: cultivation and utilization. Beiging, China.

[14] Lucas, M.; E. Martínez.; F. López; M. Abellán; F. García. 2011. El cultivo forestal de Paulownia spp: Primeros resultados de si aplicación en Castilla La Mancha. Universidad de Castilla La Mancha, Escuela superior de Ingenieros Agrónomos de Albacete y Departamento de Ciencias y tecnología Agroforestal y Genética.

[15] Gutiérrez, J.; Ocaña R. 2009. Manual para el Cultivo de Paulownia elongata. Disponible en: http://www.uaemex.mx/SIEA/editorial/2009/09_C_422_0643.pdf

[16] Wayne, K.; Donald, G. 2004. Tree Crops for Marginal Farmland. Paulownia. Practical guide from the University of Tennessee. $31 \mathrm{pp}$.

[17] Castellanos, O.; A. Rodríguez; J. Rodríguez; B. Rodríguez. 2006. Organogénesis indirecta y enraizamiento "in vitro" de "Paulownia elongata". Redalyc 4(15):1-12

[18] Rivas, F., Alarcón, A., Espinosa, C., Carrillo, F., Villamarín, D. (2005). Formaciones vegetales en el Ecuador, Escuela Politécnica del Ejército, Facultad de Ciencias Aplicadas.Pichincha --Ecuador.: Escuela de Ingeniería en Biotecnología Sangolquí.

[19] Centeno, M. 1993. Inventario Nacional de Plantaciones Forestales de Nicaragua. 85 p.

[20] ADEFOR (Asociación de Desarrollo Forestal y Agropecuario de Occidente). 1995. Comportamiento de 25 procedencias de 3 especies forestales del género Eucalyptus (E. camaldulensis Dehn, E. maculata Hook. F. y E. tereticornis Sm.) En Chancay (Cajamarca, Perú). Informe de investigación № 5. 24 p.

[21] González, B; Silva, C; Salgado, O. 2008. Evaluación de la capacidad de rebrotes de dos especies arbóreas en el bosque tropical seco, Nandarola Pacífico Sur. Universidad Nacional Agraria. Managua, NI. 8(11):57-61.

[22] Reyes, F.; Membreño, J.; Chávez, Y. 2013. Evaluación de variables dasométricas de rebrotes en Eucalyptus camaldulensis Dehnh, en la finca el Plantel, Nindiri, Masaya. La Calera Revista científica. 13 (20).39-45.

[23] Flores-Pinot, D., Janeth-Sorto, T., Gutiérrez-Bardales, J., Arias-Aguilar, D., Valverde, J. C., \& Mora- Molina, J. (2018). Capacidad de rebrote de Leucaena macrophylla Benth 
con fines dendroenergéticos en Cortes, Honduras. Revista Forestal Mesoamericana Kurú, 16(38), 47-54. Doi. 10.18845/rfmk.v16i38.3995

[24] Singhala, A.; Kumarb, M.; Bhattacharyab, M.; Kumaric, N.; Kumar, P.; Devendra, J.; Chauhanalndu, K.; Thakur, S. 2018. Pretreatment of Leucaena leucocephala wood by acidified glycerol: optimization, severity index and correlation analysis, Bioresource Technology, vol. 265, pp. 214-223.

[25] Ríos, J. 2017. Evaluación del crecimiento y rendimiento de biomasa aérea en rebrotes de cepas de dos años en cultivos dendroenergéticos. Tesis para optar al grado de doctor en ciencias forestales. Universidad de Concepción-chile.

[26] Nassara, R.; Nermeen, F. T.; Redac, M. 2015. Active yeast extract counteracts the harmful effects of salinity stress on the growth of leucaena plant. Scientia Horticulturae, vol. 30, pp. 61-67.

[27] Díaz, G. 2005. Establecimiento y evaluación de cercas vivas en Pacora, San Francisco Libre. Trabajo de diploma. UNA. Managua, Nicaragua. 27 p.

[28] Reda, F.; Maximous, S.; El-Kobisy, O. 2000. Morphological and anatomical studies on leucaena (Leucaena leucocephala) plants grown under stress of different levels of salinity in irrigation water. Bull. Fac. Agric. Cairo Univ, vol. 4, pp. 309-330.

[29] Plaza del Pino, J. 2011. Evaluación de la capacidad de rebrote en clones híbridos de Populus destinados a la producción de biomasa para energía. Trabajo fin de carrera Escuela Universitaria de Ingeniería Técnica Forestal. Universidad Politécnica de Madrid. 140 pp

[30] Guilcapi, D. 2015. Estudio de adaptabilidad de tres especies forestales, del genero Paulownia ( $P$. fortunei, $P$ elongata e Hibrido entre $P$. fortunei ${ }^{*} P$. elongata). A las condiciones de sitio estepa espinosa de Tunshi, Cantón Riobamba, Provincia de Chimborazo. Tesis para optar al grado de Ingeniero Agrónomo. Facultad de Recursos Naturales. Escuela Superior Politécnica de Chimborazo-Ecuador.106 pp.

[31] Geldres, E.; Schlatter. J.; Marcoleta, A. 2004. Monte Bajo, opción para tres especies de Eucaliptos en segunda rotación, un caso en la provincia de Osorno, Décima Región, Chile. Revista Bosque 25(3) 57-62

[32] Salgado, O.; Silva, Z. 2008. Evaluación de la capacidad de rebrote de dos especies arbóreas del bosque seco secundario de Nandarola, Nandaime, Granada. Ingeniería thesis, Universidad Nacional Agraria, UNA.

[33] Guzmán, L. 2015. Estudio de adaptabilidad de tres especies forestales, del genero Paulownia ( $P$. fortunei, $P$. elongata e Hibrido entre $P$. fortunei ${ }^{*} P$. elongata). A las condiciones de sitio Bosque húmedo Tropical de la Estación INIAP-Pichilingue, Cantón Quevedo, provincia de los Ríos. Tesis para optar al grado de Ingeniero 
Agrónomo. Facultad de Recursos Naturales. Escuela Superior Politécnica de Chimborazo-Ecuador.105 pp. 